\title{
Análisis de los efectos de los "equipos de aprendizaje por divisiones de rendimiento" en el aprendizaje y los resultados académicos
}

\author{
Analyzing "Student Team-Achievement Divisions" Effects in Learning and Academic Results

\section{Análise dos efeitos das "Equipes de aprendizagem por divisões de desempenho" na aprendizagem e os resultados acadêmicos}

Sandra Vázquez-Toledo

Universidad de Zaragoza

Facultad de Ciencias Humanas y Educación

Huesca, España

svaztol@unizar.es

https://orcid.org/0000-0003-2206-2299

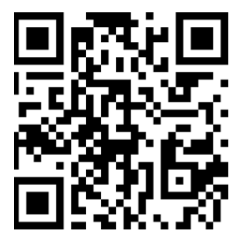

Cecilia Latorre-Cosculluela

Universidad de Zaragoza

Facultad de Ciencias Humanas y Educación

Huesca, España

clatorre@unizar.es

iD https://orcid.org/0000-0002-6083-8759

Marta Liesa-Orús

Universidad de Zaragoza

Facultad de Ciencias Humanas y Educación

Huesca, España

martali@unizar.es

iD https://orcid.org/0000-0002-9685-8399

Recibido • Received • Recebido: 17 / 02 / 2019

Corregido • Revised • Revisado: 02 / 08 / 2020

Aceptado • Accepted • Aprovado: $19 / 11 / 2020$

\begin{abstract}
Resumen: En el ámbito universitario se exige un tipo de respuesta a las necesidades formativas de las futuras personas docentes fundamentada en una transformación metodológica en la que sea el estudiantado el centro de su proceso de aprendizaje. A la luz de dicha necesidad, se propuso este estudio en aras de desarrollar una experiencia de aprendizaje cooperativo con estudiantes del Grado de Magisterio, apostándose por una de las estrategias enmarcadas en estos métodos de aprendizaje: los equipos de aprendizaje por divisiones de rendimiento (STAD). Como segundo objetivo, se propone analizar las percepciones sobre la eficacia de la aplicación de este procedimiento en el aprendizaje y en el desarrollo de competencias profesionales. A partir de un estudio descriptivo con una metodología mixta, se seleccionó una muestra de participantes conformada por un total de 123 estudiantes de esta titulación universitaria, a quienes se les administró un cuestionario de valoración diseñado ad hoc y validado mediante juicio experto. Posteriormente, se realizaron dos grupos de discusión que
\end{abstract}


http://doi.org/10.15359/ree.25-1.1

http://www.una.ac.cr/educare

educare@una.ac.cr

permitieran complementar la información recabada en la primera fase de la investigación. Los resultados obtenidos y posteriormente sometidos a análisis permiten concluir evidentes mejoras relacionadas con el proceso de aprendizaje del estudiantado universitario, con los estados motivacionales y las actitudes positivas, con el desarrollo de competencias transversales $y$, finalmente, con los resultados académicos. Considerando estas mejoras reflejadas, se recomienda que cada docente adapte, en cualquier caso, su modo de enseñar a las características de su grupo y al modo en que sus estudiantes aprenden.

Palabras claves: Educación superior; aprendizaje cooperativo; percepciones; rendimiento.

Abstract: A type of response to future teachers' educational needs, based on a methodological transformation in which the students are the protagonists of their learning process, is required in the university field. In light of these needs, this study was proposed to develop a cooperative learning experience with students of the Teaching Degree, betting on one of the strategies framed in these learning methods: the Student Team-Achievement Divisions (STAD). As a second objective, it was proposed to analyze the perceptions of the effectiveness of applying this procedure in learning and the development of professional skills. Based on a descriptive study with a mixed methodology, a sample of 123 students from this university degree was selected; they were administered an assessment questionnaire designed ad hoc and validated by an expert judgment. Subsequently, two discussion groups were held to complement the information collected in the first phase of the research. The obtained results, and later submitted to descriptive analysis, allowed to conclude evident improvements related to the university students' learning process, to the motivational states and positive attitudes, to the development of transversal competencies, and, finally, to academic results. As these improvements are considered, it is recommended that each teacher adapts, in each case, their way of teaching to the characteristics of their group and how their students learn.

Keywords: Higher education, cooperative learning, perceptions, performance.

Resumo: No ambiente universitário, é necessário um tipo de resposta às necessidades de treinamento de futuros professores, com base em uma transformação metodológica em que o corpo discente é o centro de seu processo de aprendizagem. Diante dessa necessidade, este estudo foi proposto com o objetivo de desenvolver uma experiência de aprendizagem cooperativa com os alunos do Curso de Licenciatura, apostando em uma das estratégias enquadradas nesses métodos de aprendizagem: as Equipes de Aprendizagem por Divisões de Desempenho (STAD). Como segundo objetivo, propõe-se analisar as percepções sobre a eficácia da aplicação desse procedimento na aprendizagem e no desenvolvimento de habilidades profissionais. A partir de um estudo descritivo com metodologia mista, foi selecionada uma amostra de participantes, composta por um total de 123 estudantes desse curso universitário, aos quais foi aplicado um questionário de avaliação elaborado ad hoc e validado por julgamento de especialistas. Posteriormente, dois grupos de discussão foram realizados para complementar as informações coletadas na primeira fase da investigação. Os resultados obtidos e posteriormente submetidos à análise permitem concluir melhorias evidentes relacionadas ao processo de aprendizagem do corpo discente da universidade, com estados motivacionais e atitudes positivas, com o desenvolvimento de competências transversais e, finalmente, com resultados acadêmicos. Considerando essas melhorias refletidas, recomenda-se que cada professor adapte, em qualquer caso, sua maneira de ensinar às características de seu grupo e à maneira como aprende.

Palavras-chave: Ensino superior; aprendizagem cooperativa; perceções; desempenho.

Sandra Vázquez-Toledo, Cecilia Latorre-Cosculluela y Marta Liesa-Orús

Los artículos de la Revista Electrónica Educare del Centro de Investigación y Docencia en Educación de la Universidad Nacional, Costa Rica, se comparten bajo términos de la Licencia Creative Commons: Reconocimiento, No Comercial, Sin Obra Derivada 3.0 Costa Rica. Las autorizaciones adicionales a las aquí delimitadas se pueden obtener en el correo: educare@una.cr 


\section{Introducción}

La educación va, progresivamente, desplazándose hacia modelos colaborativos y cooperativos deenseñanza-aprendizaje centrados en el estudiantado. Estos buscan su implicación activa en las actividades y, precisamente, el aprendizaje cooperativo es la metodología que tiene mayor cabida en estos retos educativos (González y Díaz Matajira, 2005). Hasta la fecha, numerosa evidencia empírica ha demostrado las consecuencias favorables que tiene esta metodología de aprendizaje cooperativo dentro de las aulas, tanto en de edades tempranas de la niñez (Reese et al., 1995) como con estudiantado universitario (Barton et al., 2018; Ravenscroft et al., 1995). El aprendizaje cooperativo es una metodología activa que, cada vez con mayor habitualidad, es implementada dentro de las aulas universitarias respondiendo, entre otras, a las demandas del Espacio Europeo de Educación Superior (Barton et al., 2018; Šerić y Praničević, 2018). De hecho, la competencia para trabajar en equipo se alza, desde hace ya varios años y todavía en nuestros días, como una de las técnicas más ampliamente adoptadas en los procesos de formación de cualquier nivel educativo (Putpuek y Kiattikomol, 2017; Puzio y Colby, 2013). Se considera, incluso, como una competencia ampliamente transferible y utilizable en el proceso de aprendizaje a lo largo de la vida y, también, en el ámbito laboral (Johnson et al., 2014).

Con el término de aprendizaje cooperativo, se engloban multitud de técnicas y métodos que encuentran sus diferencias en el grado de interdependencia de las recompensas, de la tarea, de la responsabilidad individual y de la estructura impuesta por el personal docente (Fabra Sales, 1992). Concretamente, aquellas técnicas basadas en una estructura de recompensa interdependiente, consisten en ofrecer un premio al equipo que dependerá de las recompensas individuales. En este grupo, el autor representativo y creador de las diferentes técnicas que en él se incluyen es Slavin (1978). Así, esta primera agrupación de métodos incorpora los equipos de aprendizaje por divisiones de rendimiento (Student Teams-Achievement Divisions, en adelante STAD), los torneos de aprendizaje por equipos (Teams-Games-Tournement, TGT) y el aprendizaje individual asistido por equipos (Team Assisted Individualization, TAI). Tanto en la técnica de los TGT como los STAD, constituidas como los procedimientos clásicos más aplicados dentro del aprendizaje por equipos (Student Team Learning), el alumnado trabaja con la concepción de equipo y la comparación de logros entre componentes de cada grupo (Slavin, 1978; Soto Fernández, 2009). Estos métodos subrayan la utilización de metas y recompensas de equipo y el éxito solo puede ser alcanzado si cada integrante del grupo adquiere los aprendizajes adecuadamente (Genç, 2016; León del Barco, 2002).

Entendido como un método de aprendizaje cooperativo, la técnica del STAD permite al estudiantado dominar los contenidos deseados a la par que fomenta la comunicación entre miembros del equipo, la aplicación de contenidos y la responsabilidad individual y grupal en el aprendizaje (Hunt et al., 2003). En la Tabla 1 pueden consultarse las características generales de este método. El argumento que ofreció Slavin (1978) para justificar la necesidad de implementar 
http://doi.org/10.15359/ree.25-1.1

http://www.una.ac.cr/educare

educare@una.ac.cr

en las clases este tipo de metodologías hace referencia, por un lado, a que gran cantidad de estudiantes encontraban los programas de trabajo educativo demasiado aburridos. Además, la estructura excesivamente individualizada de las tareas provocaba un aislamiento social entre estudiantes, y reducía las posibilidades de interacción y, en consecuencia, la motivación (Slavin y Oickle, 1981).

Tabla 1: Características de los STAD

\begin{tabular}{|c|c|}
\hline Número de integrantes & $4-5$ \\
\hline Estructura de la meta & $\begin{array}{l}\text { Alta: la estructura de la recompensa permite que la persecución de las } \\
\text { metas por parte del estudiantado dependa de que las demás personas } \\
\text { alcancen el éxito. }\end{array}$ \\
\hline Estructura de la tarea & $\begin{array}{l}\text { Baja: no se establece división de la tarea ni especialización por parte del } \\
\text { estudiantado en la tarea. }\end{array}$ \\
\hline Estructura de la recompensa & $\begin{array}{l}\text { Alta: se programan incentivos y premios a la cooperación basados en } \\
\text { los aprendizajes individuales de cada estudiante. Por tanto, aparece una } \\
\text { competencia intergrupal para alcanzar estas recompensas. }\end{array}$ \\
\hline Interdependencia de integrantes & $\begin{array}{l}\text { Atendiendo el tipo de estructura de la recompensa, se logra una gran } \\
\text { dependencia entre el estudiantado. }\end{array}$ \\
\hline Responsabilidad de integrantes & $\begin{array}{l}\text { Alta: viene motivada por la estructura de la recompensa. Cada estudiante } \\
\text { recibe, además, información sobre la contribución que ha realizado a las } \\
\text { puntuaciones grupales. }\end{array}$ \\
\hline Tipo de evaluación & Pruebas individuales durante 15 minutos. \\
\hline
\end{tabular}

Nota: Adaptado de León del Barco, 2002.

Mediante la utilización de este método, Slavin (1978) asigna a cada equipo cuatro o cinco integrantes. Estos grupos están constituidos por estudiantes con bajo, medio y alto rendimiento y, por supuesto, de diferentes clases sociales, culturales y étnicas. Se pretende, con ello, la búsqueda de la máxima heterogeneidad entre cada equipo a fin de que cada grupo se conforme como un "microcosmos" de toda el aula. En un primer momento, el personal docente presenta la explicación de la cuestión objeto de aprendizaje y, posteriormente, cada equipo y sus integrantes trabajan para dominar la lección. Durante este proceso, en el que se puede trabajar individualmente o en parejas, van turnándose para hacerse preguntas, discuten en grupo y utilizan cualquier otra herramienta para aprender el material. En última instancia, se realizan pruebas individuales, y los resultados de estas se traducen en puntuaciones de equipo mediante un sistema conocido como "rendimiento por divisiones". El personal docente evalúa de acuerdo con grupos de nivel homogéneos (en lugar de realizar una comparativa con el conjunto de la clase). Aquellos equipos ganadores serán los que reciban la recompensa (León del Barco, 2002). 
En definitiva, durante los últimos años la investigación empírica ha evidenciado la existencia de diferentes prácticas docentes que tienen el enorme potencial de estimular una participación más activa y comprometida de su alumnado. A consecuencia de algunos de estos procesos, y ejemplo de ello es el aprendizaje cooperativo, se logra estimular el pensamiento crítico, la autonomía y el aprendizaje de distintas competencias transversales, profesionales y actitudinales por parte del estudiantado (González González, 2014). A la par, también se fomenta la educación inclusiva, en tanto que la formación de grupos heterogéneos tiene la finalidad de atender la diversidad de características y necesidades estudiantiles (Delors, 1996). Hasta el momento, se ha destacado la participación activa como uno de los componentes principales para alcanzar un proceso de enseñanza efectivo. Precisamente por ello, se desarrolla en un aula universitaria una experiencia de aprendizaje cooperativo con estudiantes del Grado de Magisterio y se apuesta, como estrategia educativa, por los STAD. Se plantea, por tanto, un estudio empírico de corte descriptivo, con el objetivo de analizar las percepciones, del estudiantado que ha participado en la experiencia, acerca de los efectos que esta metodología ha tenido sobre su aprendizaje y el desarrollo de competencias profesionales.

\section{Método}

El estudio es de carácter descriptivo y se enmarca en un tipo de investigación con metodología mixta. De acuerdo con Creswell y Plano Clark (2007), el estudio se guiaría por la técnica de la triangulación en tanto que se pretenden combinar las ventajas de la metodología cuantitativa y cualitativa para la obtención de información que se complemente alrededor de una misma temática de estudio.

\section{Participantes}

Lamuestradeparticipantesseleccionadaestuvoconformadaporuntotal de 123 estudiantes de universidad del Grado de Magisterio de Educación Primaria (63 correspondientes al grupo A, y 60 al grupo B). La distribución en ambos grupos (A y B) responde a criterios administrativos fijados al inicio de curso por la secretaría de la facultad a la que el estudiantado acude. Del conjunto de la muestra, un $39 \%$ estaba conformada por alumnado de género masculino, mientras que el 61 $\%$ restante estaba constituido por estudiantes de género femenino, en edades comprendidas entre los 18 y 24 años. En esta ocasión, el tipo de muestreo llevado a cabo fue no probabilístico y de carácter intencional, en tanto que se pretendía recoger información de un determinado contexto. De manera similar, en una segunda fase de la investigación desarrollada mediante la realización de grupos de discusión, el procedimiento planteado también se ha servido de un tipo de muestreo intencional, para posibilitar una selección de estudiantes inicialmente con interés por participar (ver características de participantes en la Tabla 2). 
http://doi.org/10.15359/ree.25-1.1

http://www.una.ac.cr/educare

educare@una.ac.cr

Tabla 2: Características de participantes de los grupos de discusión

\begin{tabular}{ll}
\hline \multicolumn{1}{c}{ Grupo A } & \multicolumn{1}{c}{ Grupo B } \\
\hline 4 estudiantes género femenino (EF1, EF2, EF3, EF4) & 4 estudiantes género femenino (EF5, EF6, EF7, EF8) \\
3 estudiantes género masculino (EM1, EM2, EM3) & 3 estudiantes género masculino (EM4, EM5, EM6) \\
\hline Total: 7 estudiantes & Total: 7 estudiantes \\
\hline
\end{tabular}

Nota: Elaboración propia.

\section{Instrumentos}

En un primer momento, se aplicó un cuestionario ad hoc en soporte online, diseñado y construido previamente con base en una profunda revisión de la bibliografía sobre métodos de estructuración de la actividad de aula basados en equipos de aprendizaje por divisiones de rendimiento y su funcionamiento. El cuestionario estaba conformado por 11 aseveraciones de tipo Likert, con cuatro niveles de respuesta que van desde "totalmente en desacuerdo" hasta "totalmente de acuerdo". Para esta muestra, se obtuvo un coeficiente alfa de Cronbach satisfactorio, traducido en un índice de 0,82. Los datos obtenidos mediante este instrumento fueron complementados con información recabada a través de la realización de los grupos de discusión. En la segunda fase de la investigación se utilizaron los grupos de discusión para profundizar en el análisis de las percepciones del estudiantado. En estos grupos se utilizaron preguntas que tenían la finalidad de estudiar los pareceres más personales e individuales, sin las limitaciones que el cuestionario trae consigo. El desarrollo de estos grupos se llevó a cabo una vez recabados los datos del cuestionario.

\section{Procedimiento de aplicación de los STAD y análisis de datos}

En la Fase I de la planificación del aprendizaje de aula con esta metodología se lleva a cabo una presentación inicial, por la parte docente, de los objetivos a conseguir y de la técnica a aplicar. Quedaron establecidos, como objetivos primordiales, el logro de una mejora del aprendizaje y de los resultados alcanzados por el estudiantado. En esta primera fase se pretendió, además, que el estudiantado universitario trabajara eficazmente diversas competencias transversales o genéricas, que son esenciales en la profesión docente, a través del trabajo en equipo (cooperación, negociación, respeto, tolerancia, liderazgo, pensamiento crítico y creativo, escucha activa, resolución de conflictos, toma de decisiones, convivencia y el respeto, autonomía, habilidades sociales, apoyo, la confianza y aceptación). Su docente aclaró, además, el funcionamiento de los STAD, quedaron fijadas un total de cuatro pruebas cuatrimestrales en las que sus conocimientos y competencias conforman el objeto de evaluación. 
http://doi.org/10.15359/ree.25-1.1

Al finalizar el cuatrimestre, cada equipo base obtiene su recompensa en función del rendimiento alcanzado (es decir, según la calificación obtenida). La nota de las pruebas del STAD son sumadas $y$, en función de la media obtenida -es decir, dependiendo del tramo en el que quede situado el equipo, de acuerdo con la puntuación alcanzada- se obtiene dicha recompensa estructurada en intervalos de calificación (ver Tabla 3). Uno de los requisitos esenciales que se fijaron de antemano, en lo que respecta al funcionamiento de los equipos de trabajo, se vincula al control de la asistencia a las clases de la totalidad y de cada miembro del grupo. En este sentido, si alguien no acudió en tan solo una ocasión a la prueba de STAD, solamente pudo obtener la mitad de la puntuación. En aquellos casos en los que se registraron dos faltas de asistencia, directamente el estudiantado no pudo optar por la recompensa.

Tabla 3: Relación entre calificación obtenida en las pruebas en STAD y recompensa adjudicada

\begin{tabular}{cc}
$\begin{array}{c}\text { Media de las calificaciones obtenidas en las pruebas } \\
\text { en STAD }\end{array}$ & $\begin{array}{c}\text { Recompensa (en términos de aumento de la } \\
\text { calificación académica) }\end{array}$ \\
\hline $5-5,99$ & 0,1 puntos \\
$6-6,99$ & 0,15 puntos \\
$7-7,99$ & 0,2 puntos \\
$8-8,99$ & 0,25 puntos \\
$9-9,99$ & 0,3 puntos \\
10 & 0,35 puntos \\
\hline
\end{tabular}

Nota: Elaboración propia.

En la Fase II se crearon los equipos base, de tal modo que cada uno de estos grupos de entre 4-5 estudiantes quedase dispuesto como un "microcosmos" representativo de la totalidad del aula. La Fase III se refiere a la misma puesta en marcha y desarrollo de las tareas propias de estos equipos de aprendizaje. Durante un periodo previo de estudio, cada miembro reflexionaba sobre los materiales de la asignatura proporcionados por su docente, y ayudaba, además, a sus compañeros y compañeras a aprender. El alumnado resolvía dudas, se explicaban y se examinaban entre sí, buscando la preparación idónea para la prueba. Durante los 20 primeros minutos, el estudio del alumnado era de carácter individual para proseguir, una vez cumplimentado ese periodo, con un tipo de preparación de los materiales y contenidos en un formato grupal. En último lugar, para la realización de estas pruebas en STAD el estudiantado dispuso de un intervalo de tiempo entre 15 y 20 minutos.

La Fase IV del proceso de desarrollo de las pruebas basadas en los STAD se caracteriza, a grandes rasgos, por la devolución de los resultados obtenidos a cada equipo base. De este modo, los resultados alcanzados por cada grupo fueron devueltos para que tuvieran la posibilidad de reconocer sus errores y establecer propuestas de mejora de funcionamiento tanto individual 
http://doi.org/10.15359/ree.25-1.1

http://www.una.ac.cr/educare

educare@una.ac.cr

como grupal que les posibilitara un progresivo avance en sus aprendizajes. Finalmente, se procedió a una publicación de la recompensa obtenida por el grupo una vez finalizado el periodo lectivo del cuatrimestre en la Fase $V$. En la Tabla 4 se muestra una síntesis de las fases seguidas en la aplicación de la experiencia.

Tabla 4: Fases en la aplicación de la experiencia STAD

\begin{tabular}{ll}
\hline Fase I & Presentación de objetivos, técnica y funcionamiento. \\
Fase II & Creación de equipos de base. \\
Fase III & Puesta en marcha y desarrollo de tareas. \\
Fase IV & Devolución de resultados. \\
Fase V & Publicación de recompensas. \\
\hline
\end{tabular}

Nota: Elaboración propia.

Posteriormente a la puesta en marcha de todas las fases descritas, se fijó el procedimiento a seguir en el desarrollo del estudio empírico. En una primera etapa, y tras la realización del juicio de personas expertas para validar el instrumento de recogida de datos cuantitativos, se llevó a cabo la distribución (a través del correo institucional de la universidad del que cada estudiante dispone) y cumplimentación online de estos cuestionarios entre el estudiantado que conformaba la muestra. Por otro lado, durante la fase preparatoria de la realización de los grupos de discusión, se revisaron las publicaciones e investigaciones que, hasta el momento, se encontraban disponibles en materia de utilización de esta metodología cooperativa en las aulas universitarias. Tomandolas como referencia, se dispusieron las preguntas abiertas que guiarían el desarrollo del discurso en ambos grupos. Con la finalidad de asegurar una fiabilidad en el desarrollo de las sesiones de recogida de información en estos grupos focales, fue la misma moderadora la responsable de orientar el proceso en ambos.

En la primera fase de la investigación, en la que la recogida de datos se realizó utilizando el cuestionario, la recolección de la información se llevó a cabo en un soporte online, mientras que los grupos de discusión se realizaron presencialmente en las aulas ordinarias en las que estos grupos de estudiantes habitualmente cursan la asignatura en la que esta experiencia de investigación se enmarca. Todas las personas encuestadas fueron informadas de antemano de la voluntariedad en cuanto a la participación en el estudio y de la no repercusión que esta fase empírica de recogida de datos tenía sobre el desarrollo del curso de la asignatura. Finalizada la cumplimentación de la encuesta, se dio a las personas participantes la posibilidad de realizar cualquier comentario y de consultar todas las dudas que considerasen oportunas al respecto.

En lo que respecta al análisis de datos procedentes de la administración del cuestionario, se realizaron estadísticos descriptivos (en términos de media, desviaciones típicas y frecuencias). Por su parte, para el análisis de la información recabada con los grupos de discusión se consideró 
http://doi.org/10.15359/ree.25-1.1

la propuesta de Huberman y Miles (2000), compuesta por tres subprocesos vinculados entre sí. Este proceso se inició con la recogida de información que, en este estudio, correspondió a la realización de los grupos de discusión. Posteriormente, se llevó a cabo su reducción, esto es, se seleccionó, clasificó y codificó la información atendiendo a las categorías de análisis. Tras ello, se procedió a la extracción e interpretación de las conclusiones tomando como referencia la perspectiva planteada en el marco teórico inicial. En esta fase, se utilizó la comparación y la triangulación como procedimientos para asegurar la validez de la información.

\section{Resultados}

En la Tabla 5 aparecen los estadísticos descriptivos de las medias y las desviaciones típicas para cada uno de los ítems del cuestionario que la muestra de estudiantes ha respondido. En su conjunto, el estudiantado se posiciona más de acuerdo con el hecho de que los STAD han influido de forma positiva en la calificación obtenida en el examen final y de que, gracias a ello, ha aumentado su compromiso de trabajo con el equipo. Destaca, con una tasa menor de acuerdo, la oportunidad que los STAD les ha dado para compartir apuntes, y el hecho de que su comprensión ante los contenidos haya aumentado comparándola con la comprensión que habrían tenido estudiando solamente de manera individual.

Tabla 5: Estadísticos descriptivos: Medias y D. T.

\begin{tabular}{|c|c|c|}
\hline & Media & Desv. típ. \\
\hline Los STAD han influido de manera positiva en la nota del examen final. & 1,67 &, 561 \\
\hline Gracias a los STAD he estudiado antes de lo que hubiera hecho. & 1,82 & ,757 \\
\hline He solventado dudas de contenido con mis compañeros y compañeras. & 1,93 & ,745 \\
\hline He compartido apuntes. & 1,96 & ,860 \\
\hline Me ha ayudado a autorregularme (planificarme mejor). & 1,94 & 694 \\
\hline $\begin{array}{l}\text { He comprendido contenido que no hubiera conseguido comprender estudiando solo } \\
\text { de manera individual. }\end{array}$ & 2,00 & 823 \\
\hline $\begin{array}{l}\text { Ha aumentado el interés por esta asignatura gracias a la realización de los STAD y a otras } \\
\text { estrategias de aprendizaje cooperativo. }\end{array}$ & 2,01 & 749 \\
\hline He entendido mejor los temas difíciles. & 1,91 &, 570 \\
\hline $\begin{array}{l}\text { He mejorado mis habilidades comunicativas: comprender, explicar, preguntar y } \\
\text { responder, debatir, utilizar correctamente terminología de la asignatura, etc. }\end{array}$ & 1,85 & 657 \\
\hline Mi compromiso con el equipo se ha incrementado por los STAD. & 1,76 & ,725 \\
\hline $\begin{array}{l}\text { Han mejorado mis habilidades sociales (empatía, negociación, escucha, asertividad, } \\
\text { resolución de conflictos, autocontrol, etc.) participando en los STAD. }\end{array}$ & 1,85 & 657 \\
\hline
\end{tabular}

Nota: Elaboración propia. 
http://doi.org/10.15359/ree.25-1.1

http://www.una.ac.cr/educare

educare@una.ac.cr

Por otro lado, en la Tabla 6 se muestran las frecuencias de respuesta de cada una de las cuatro opciones que la muestra de estudiantes ha indicado. A grandes rasgos, se aprecia una tendencia mayoritaria a entender, desde una visión positiva, los efectos que para su aprendizaje y compromiso tiene este modo de trabajo en el aula universitaria. En esta ocasión, existe un porcentaje relativamente elevado de estudiantes que considera que los STAD no le han ayudado a autorregularse (20,9\%), y que su interés por la asignatura no se ha visto considerablemente aumentado gracias a la implementación de esta metodología de aprendizaje cooperativo $(25,4 \%)$.

Tabla 6: Estadísticos descriptivos: Frecuencias

\begin{tabular}{lcccccccc}
\hline & $\begin{array}{c}\text { Totalmente } \\
\text { de acuerdo }\end{array}$ & $\%$ & $\begin{array}{c}\text { De } \\
\text { acuerdo }\end{array}$ & $\%$ & En desacuerdo & $\%$ & $\begin{array}{c}\text { Totalmente en } \\
\text { desacuerdo }\end{array}$ & $\%$ \\
\hline Ítem 1 & 25 & 37,3 & 39 & 58,2 & 3 & 4,5 & 0 & 0 \\
Ítem 2 & 24 & 35,8 & 33 & 49,3 & 8 & 11,9 & 2 & 3 \\
Ítem 3 & 19 & 28,4 & 36 & 53,7 & 10 & 14,9 & 2 & 3 \\
Ítem 4 & 22 & 32,8 & 30 & 44,8 & 11 & 16,4 & 4 & 6 \\
Ítem 5 & 18 & 26,9 & 35 & 52,2 & 14 & 20,9 & 0 & 0 \\
Ítem 6 & 18 & 26,9 & 34 & 50,7 & 10 & 14,9 & 4 & 6 \\
Ítem 7 & 17 & 25,4 & 33 & 49,3 & 16 & 23,9 & 1 & 1,5 \\
Ítem 8 & 14 & 20,9 & 45 & 67,2 & 8 & 11,9 & 0 & 0 \\
Ítem 9 & 19 & 28,4 & 40 & 59,7 & 7 & 10,4 & 1 & 1,5 \\
Ítem 10 & 26 & 38,8 & 31 & 46,3 & 8 & 11,9 & 1 & 15 \\
Ítem 11 & 18 & 26,9 & 43 & 64,2 & 4 & 6 & 2 & 3 \\
\hline
\end{tabular}

Nota: Elaboración propia.

Siguiendo los procedimientos de análisis cualitativos indicados en la sección previa, los datos recabados llevan a la extracción de un conjunto de resultados que evidencian que, gracias a la puesta en marcha de estrategias metodológicas de estas características, pueden lograrse mejoras respecto a cuatro aspectos generales: progresos en cuanto al proceso de aprendizaje, mejoras en la motivación y actitudes positivas adoptadas ante los aprendizajes, avances en cuanto al desarrollo de competencias transversales provenientes de la estructura de trabajo en equipo y, finalmente, mejoras en el logro de resultados académicos.

Los avances registrados en cuanto al proceso de aprendizaje provienen, en primer lugar, del aprendizaje entre iguales. Boud y Solomon (2001) definen el aprendizaje entre iguales como situaciones educativas en las cuales el estudiantado tiene oportunidades recíprocas de aprender 
http://doi.org/10.15359/ree.25-1.1

y enseñar, es decir, de aprender de las otras personas y con estas. Durante el estudio colectivo llevado a cabo en la realización de estas pruebas en STAD, el alumnado comparte apuntes, resuelve dudas y se explican el contenido entre sí. En consecuencia, se ayudan mutuamente en tanto que el éxito individual depende del éxito grupal, y viceversa.

A este respecto, la información recabada en los grupos de discusión permitió concluir que, con la implantación de esta metodología, el conjunto de participantes comenzó a estudiar con más antelación de lo que habitualmente lo hacen en otras asignaturas. Además, alcanzaron una íntegra comprensión de los contenidos presentados gracias a la ayuda y explicaciones que, mutuamente, recibieron por parte de pares. Una parte llegó incluso a manifestar una mayor comprensión de los conceptos cuando eran sus iguales de equipo quienes les ofrecían aclaraciones al respecto.

Al hacer la prueba de STAD, he visto su efectividad en cuanto a la cooperación del grupo y el estudio de la asignatura. (EF4)

Una interesante forma de aprender el contenido de manera más dinámica y con el apoyo de tus compañeros. (EF8)

Al hacer los STAD en grupo (Equipo Base), y teniendo en cuenta que los cuatro componentes pensamos lo mismo, es más probable que lleguemos a la respuesta correcta antes que de manera individual. (EM3)

Del análisis de los datos recabados tras la administración del cuestionario, se desprende que un $82 \%$ del estudiantado encuestado consideró que, a causa del compromiso personal que estas tareas les suponen, ha logrado solventar dudas de contenido con sus pares de la universidad. De este modo, un $78,8 \%$ del conjunto del alumnado indicó el logro de una comprensión de determinados contenidos de la asignatura que, con una alta probabilidad, no habría conseguido resolver de manera individual. Gracias a la utilización de este método de aprendizaje, un significativo $88,8 \%$ de quienes participaron afirmaron haber entendido considerablemente mejor aquellas cuestiones más complejas o que conllevaban una dificultad más elevada. De forma complementaria, entre miembros de los equipos se ha logrado fomentar también la tarea de compartir los diversos materiales de estudio, reflejo de lo cual un 77,6 \% se ha visto comprometido con ello.

Como segundo avance reconocido en lo que respecta al proceso de aprendizaje implícito en este método de organización de los procesos de enseñanza en el aula universitaria, se destaca la autorregulación de sus propios ritmos de trabajo. Así, se ofreció con ello al estudiantado la posibilidad de verificar sus aprendizajes, estableciendo comparativas entre sus resultados 
http://doi.org/10.15359/ree.25-1.1

http://www.una.ac.cr/educare

educare@una.ac.cr

actuales con rendimientos anteriores a través del feedback que su docente les ha devuelto. Este procedimiento les permite planificar mejor su proceso de aprendizaje y tomar decisiones pertinentes al respecto, tanto a nivel grupal como individual.

Mediante la recogida de datos cualitativos a través de los grupos de discusión, ha podido destacarse la utilidad del método de aprendizaje cooperativo basado en los STAD, en cuanto a la organización que el estudiantado realiza de su propio estudio. Un 85,1 \% del conjunto de estudiantes declaró haber iniciado sus tareas relacionadas con el estudio de la materia antes de lo que, en circunstancias habituales, lo hubieran hecho. Un 79,1 \% confirmó que el compromiso con los STAD le ha ayudado a autorregularse en su propio aprendizaje. Durante la puesta en marcha de los grupos focales, pudo concluirse también la eficacia de esta técnica en tanto que ha posibilitado a este alumnado universitario una más profunda e intensa preparación del examen final de la asignatura, así como una progresiva organización e inversión de esfuerzos en el estudio.

La realización de los STAD me ha parecido una buena actividad para mejorar nuestro estudio, ya que nos permite estudiar los contenidos sin dejarlos para el final y, a la hora de estudiar antes del examen definitivo, todo resulta conocido y es mucho más fácil estudiar. (EF1)

Los STAD son muy positivos, ya que ayudan a mentalizarnos que debemos estudiar poco a poco y no dejarlo todo para cuando se acerque el examen final. (EM2)

Me parece muy bien que se realicen los STAD, ya que nos ayuda a ir estudiando día a día. Muchos venimos de Bachiller, donde teníamos más seguimiento que en la Universidad, y con este método hemos ido estudiando día a día y también nos han ayudado a hacernos una idea de cómo pueden ser los exámenes. (EF7)

Los avances alcanzados en cuanto al proceso de aprendizaje del estudiantado universitario se han visto reflejados, también, en evidentes progresos en cuanto al compromiso y la responsabilidad individual y grupal. En este sentido, el estudiantado expuso que la metodología basada en los STAD ha logrado despertarles un sentimiento mayor de pertenencia al grupo, en tanto que su motivación se focalizaba en la obtención de una mayor recompensa que dependía tanto del esfuerzo y trabajo de cada quien, como del grupo en su conjunto. Un significativo 86,4 $\%$ del alumnado ha manifestado una opinión favorable respecto del aumento de su compromiso personal con las tareas de estudio dispuestas en su equipo base.

Pienso que es una buena forma de que el equipo base se una más (aunque siempre hay excepciones) pues, como mínimo, te tienes que leer el temario para poder aportar respuestas correctas en el examen. (EF2) 
http://doi.org/10.15359/ree.25-1.1

Un segundo ámbito de progreso en el que las mejoras detectadas han sido más que considerables, se relaciona con los estados motivacionales y actitudes positivas manifestadas por el conjunto de participantes de la investigación. En términos generales, se registraron avances en la motivación de logro, en tanto que el estudiantado apreció un evidente progreso personal que se vio reflejado en los resultados académicos finales. Utilizando los datos como evidencia empírica, quedaron confirmados los beneficios percibidos por el alumnado, con un $58,2 \%$ que resaltaron como "útiles" los STAD, y un 37,3\% que los puntuó como "muy útiles" para su aprendizaje. Un $74,7 \%$ de la muestra de participantes indicó que, gracias a la realización de estas pruebas y otras estrategias cooperativas llevadas a cabo en el aula, su interés por la asignatura ha aumentado.

Los STAD me parecen una buena manera de organizarte el temario para estudiarlo con tiempo durante el cuatrimestre $y$, personalmente, me han hecho llegar al examen de enero más segura y sin miedo. (EF6)

Es una herramienta muy productiva que me ha ayudado a sacar un mejor rendimiento y a tener mucha más motivación ante el estudio. (EF5)

Una novedosa y motivante manera de estudiar. (EM1)

Son muy útiles porque a la hora de realizar el examen ya te suena todo de haberlo estado trabajando previamente. (EM2)

Otro de los progresos evidenciados tras la recogida de datos con ambos instrumentos (cuestionario de valoración y grupo de discusión) se vincula con el desarrollo de competencias transversales provenientes de la estructura del trabajo en equipo tales como el fomento de habilidades sociales, de la capacidad de negociación, de las habilidades comunicativas y de la capacidad de liderazgo, entre algunas otras. Durante los grupos de discusión, el alumnado participante advirtió que la realización de tareas en equipo les exige mucho más esfuerzo e implicación que el trabajo individual, especialmente cuando tienen que llegar a acuerdos y a concordar la tomar decisiones.

Como aspecto positivo, han expresado haber aprendido a trabajar con pares a quienes inicialmente no conocían, lo que desarrolló así habilidades de escucha (un 88, 1 \% de las personas participantes experimentaron mejoras en cuanto a sus habilidades comunicativas, tales como la comprensión, la enunciación de preguntas y respuestas, la iniciación y mantenimiento de debates o la utilización de una terminología adecuada en el marco de desarrollo de la asignatura), empatía, negociación y gestión de la carga de trabajo y responsabilidad dentro de los equipos base. Prácticamente la totalidad del estudiantado $(91,1 \%)$ estuvo completamente de acuerdo 
http://doi.org/10.15359/ree.25-1.1

http://www.una.ac.cr/educare

educare@una.ac.cr

con la repercusión de este método de aprendizaje cooperativo en la mejora de sus habilidades sociales (más específicamente, de todas aquellas relacionadas con la asertividad, resolución de conflictos y autocontrol).

En último lugar, el proceso de recogida y análisis de datos ha permitido confirmar, tal y como se estimaba, que el método de trabajo en el aula universitaria basado en los STAD posibilita notables avances y progresos en cuanto al logro de unos resultados académicos más satisfactorios (traducido en una mejora de la calificación académica). Así pues, tan solo un $17,8 \%$ del estudiantado no obtuvo recompensa (en términos de aumento de su calificación) al finalizar el cuatrimestre, por no alcanzar una nota media en las pruebas en STAD superior a 5. En más de la mitad de los equipos base (56,2\%), la nota media obtenida fue superior a 7 . En consecuencia, a los miembros de dichos equipos se les añadió entre 0,2 y 0,35 puntos a su nota individual alcanzada en la asignatura.

Analizando los datos y la información derivada de la calificación final, durante el primer año académico en que esta experiencia fue desarrollada -curso 2015/16- se detectó tan solo un $4,7 \%$ de estudiantes que, al finalizar el curso, no superaron la asignatura. Desde ese año en que se inició una estructuración del trabajo de aula universitaria basado en STAD, el porcentaje de estudiantado suspenso ha disminuido notablemente, aspecto que puede visiblemente apreciarse en la Tabla 7 al establecer comparativas con cursos previos. También la tasa de éxito y rendimiento ha aumentado desde que esta estrategia se ha puesto en marcha en el aula. Cabe mencionar que la tasa de éxito fue entendida como la relación porcentual entre el número total de créditos superados por el alumnado en un estudio y el número total de créditos presentados a examen. Por su parte, la tasa de rendimiento quedó concretada como la relación porcentual entre el número total de créditos ordinarios superados por el estudiantado en un determinado curso académico y el número total de créditos ordinarios matriculados.

Tabla 7: Tasas de éxito y rendimiento por cursos académicos

\begin{tabular}{ccccccccccccc}
\hline Curso & $\begin{array}{c}\text { No } \\
\text { presentados }\end{array}$ & $\%$ & Sus & $\%$ & Apr & $\%$ & Not & $\%$ & Sob & $\%$ & $\begin{array}{c}\text { Tasa } \\
\text { éxito }\end{array}$ & $\begin{array}{c}\text { Tasa } \\
\text { rendimiento }\end{array}$ \\
\hline $14 / 15$ & 4 & 3,4 & 13 & 11,1 & 59 & 50,4 & 38 & 32,5 & 0 & 0 & $\mathbf{8 8 , 5}$ & $\mathbf{8 5 , 5}$ \\
$15 / 16$ & 6 & 4,7 & 6 & 4,7 & 55 & 42,6 & 54 & 41,9 & 3 & 2,3 & $\mathbf{9 5 , 1}$ & $\mathbf{9 0 , 7}$ \\
$16 / 17$ & 6 & 4,8 & 3 & 2,4 & 47 & 37,9 & 60 & 48,4 & 3 & 2,4 & $\mathbf{9 7 , 4 6}$ & $\mathbf{9 2 , 7 4}$ \\
$17 / 18$ & 6 & 4,9 & 4 & 3,3 & 42 & 34,4 & 66 & 54,1 & 0 & 0,0 & $\mathbf{9 6 , 5 5}$ & $\mathbf{9 1 , 8 0}$ \\
\hline
\end{tabular}

Nota: Elaboración propia. 


\section{Conclusiones y discusión}

Además de presentar la descripción de una experiencia de aprendizaje con la técnica de los STAD en el aula universitaria, el estudio aquí descrito, fundamentado en un tipo de metodología mixta, tenía como objetivo analizar, en términos estadísticos y desde una perspectiva más en profundidad, las percepciones del estudiantado respecto de los beneficios que este sistema les reportaba. A grandes rasgos, la puesta en marcha de este estudio ha permitido constatar que la técnica de los STAD tiene un impacto significativo en el aprendizaje estudiantil. Con esta metodología de enseñanza-aprendizaje, la juventud universitaria percibe los contenidos desde una perspectiva social en lugar de recibirlos de manera aislada, y comienza a sentirse miembros, en participación activa, de un equipo en el que tienen responsabilidades tanto individuales como grupales. En investigaciones como las de Wanzek et al. (2014) y Yusuf et al. (2015), se hace visible la oportunidad que tienen los sectores jóvenes para considerar su propio pensamiento y el de sus compañeros y compañeras de equipo y lograr, consecuentemente, una mayor comprensión de los contenidos de aprendizaje. Además, estudios como el realizado por Ginsburg-Block et al. (2006) señalan una correlación positiva entre resultados académicos y aprendizaje entre iguales.

Los resultados aquí encontrados son coincidentes con los hallazgos de previos estudios que se han llevado a cabo en contextos universitarios. Así, Slavin (1977, citado por Slavin y Oickle, 1981) demostró que, aquel estudiantado que trabajó con esta metodología de aprendizaje cooperativo, obtuvo mejoras más notables que el grupo control en cuanto a logros académicos. Y, de igual modo y tras aplicar una metodología de aprendizaje cooperativo basada en una estructura de STAD, recientemente Masran y Azizi (2018) evidencian visibles mejoras en alumnado de la etapa de Educación Primaria sobre el ámbito de su interés ante el aprendizaje, entusiasmo y fomento de habilidades sociales. Los resultados son también apoyados por otras investigaciones internacionales que avalan la eficacia de los STAD para la mejora de numerosas áreas del desarrollo del estudiantado en diferentes niveles educativos (Putpuek y Kiattikomol, 2017).

Ha quedado ampliamente demostrado que las estructuras de trabajo dentro de las aulas fundamentadas en el aprendizaje cooperativo tienen una enorme influencia sobre las interacciones que se establecen entre estudiantes. Además de ello, se ven también reforzadas otras variables tales como el rendimiento académico y la autoestima, el desarrollo de habilidades sociales, y la promoción de normas tales como la tolerancia, la solidaridad y el dominio de los estímulos impulsivos (León del Barco et al., 2014; Ovejero, 1990). De hecho, en recientes investigaciones desarrolladas en el contexto español (Mendo-Lázaro et al., 2018), se reportan también evidentes beneficios de la aplicación de metodologías de aprendizaje cooperativo en el proceso de formación de docentes, especialmente vinculadas a una mejora de habilidades sociales de los sujetos integrantes de los equipos de trabajo. En comparación con otras metodologías de instrucción directa, los STAD como método de enseñanza mejoran 
http://doi.org/10.15359/ree.25-1.1

http://www.una.ac.cr/educare

educare@una.ac.cr

notablemente el aprendizaje, a todos los niveles, del alumnado, incluso también en áreas de estudio universitario tales como economía y empresa (Van Wyk, 2015).

No cabe duda alguna de la necesidad de que el profesorado enseñe a sus estudiantes, de manera explícita, cómo proporcionar ayuda a otros compañeros y compañeras para poder aprovechar, de este modo, los beneficios que ofrece la interacción social durante las discusiones en pequeños grupos (Gillies y Khan, 2009). Además de enseñar al estudiantado a dar y recibir ayuda de las demás personas, el docente o la docente debe implicarse activamente en las discusiones que se generan dentro de este trabajo cooperativo (Reznitskaya et al., 2007). De manera similar a lo que apunta Slavin (1991), en esta experiencia el estudiantado del Grado de Magisterio valora a su docente como profesional que dispone de recursos e información valiosa, es decir, consideran más su rol de entrenamiento y guía que como autoridad.

El método de aprendizaje cooperativo aquí descrito supone un esfuerzo considerable de trabajo extra para el profesorado, así como la necesidad de establecimiento de cauces de colaboración y coordinación entre el equipo de docentes responsables de la materia. A todo ello, se le añade la controversia que, en algunos de los últimos estudios desarrollados con poblaciones de estudiantado universitario, se encuentra al respecto de la preferencia de trabajar en formato de equipos de aprendizaje cooperativo. De hecho, en el trabajo de Šerić y Praničević (2018), el estudiantado de una universidad de Croacia alude a la falta de igualdad, de respeto entre integrantes de los equipos y de consideración de las opiniones propias, como algunos de los riesgos principales de estas estructuras de aprendizaje en el aula universitaria.

Independientemente de estas cuestiones, cuando el estudiantado trabaja junto, de forma cooperativa, los resultados se traducen en evidentes mejoras de su autoestima y competencias sociales mediante la instauración de relaciones de interacción, el apoyo mutuo y la toma en consideración de las perspectivas y opiniones de los demás sujetos (Johnson y Johnson, 1999; Munir et al., 2018). Cabe apuntar que no existe un único método de enseñanza con el que lograr los mejores resultados en el ámbito universitario, sino que, de acuerdo con lo señalado por García-Ruiz y González Fernández (2013), es labor de cada docente adaptar su manera de enseñar a las características de su grupo de estudiantes y a su forma de aprender. Además, el estudio aquí presentado pone de relevancia cómo un tipo de estructura de trabajo en formato cooperativo, en la que sus logros individuales les sean reconocidos, posibilita que el alumnado ejercite sus habilidades interpersonales e intrapersonales. Finalmente, las percepciones del estudiantado analizadas en este trabajo reflejan, de un modo implícito, avances en cuanto al refuerzo de la competencia para aprender a aprender en tanto que han afirmado ser capaces de desarrollar estrategias más efectivas de aprendizaje. Dicha competencia se deriva, en gran parte, de la responsabilidad individual y de la capacidad para ser personas autónomas en sus tareas de aprendizaje. 


\section{Declaración de Material complementario}

Este artículo tiene disponible como material complementario:

-La versión preprint del artículo en https://doi.org/10.5281/zenodo.3384270

\section{Referencias}

Barton, G., Bruce, A. y Schreiber, R. (2018). Teaching nurses teamwork: integrative review of competency-based team training in nursing education. Nurse Education in Practice, 32, 129-137. https://doi.org/10.1016/j.nepr.2017.11.019

Boud, D. y Solomon, N. (Eds.). (2001). Work-based learning. A new higher education? Open University Press.

Creswell, J. W. y Plano Clark, V. L. (2007). Designing and conducting mixed methods research. Sage.

Delors, J. (Preside). (1996). La educación encierra un tesoro. Informe a la UNESCO de la Comisión Internacional sobre la Educación para el siglo XXI. Santillana.

Fabra Sales, M. L. (1992). El trabajo cooperativo: Revisión y perspectivas. Aula de Innovación Educativa, 9, 5-12.

García-Ruiz, M. R. y González Fernández, N. (2013). El aprendizaje cooperativo en la universidad. Valoración de los estudiantes respecto a su potencialidad para desarrollar competencias. Revista Iberoamericana para la Investigación y el Desarrollo Educativo, 4(7), 106-128. https:// www.ride.org.mx/index.php/RIDE/issue/view/9

Genç, M. (2016). An evaluation of the cooperative learning process by sixth-grade students. Research in Education, 95(1), 19-32. https://doi.org/10.7227/RIE.0018

Gillies, R. M. y Khan, A. (2009). Promoting reasoned argumentation, problem - solving and learning during small - group work. Cambridge Journal of Education, 39(1), 7-27. https:// doi.org/10.1080/03057640802701945

Ginsburg-Block, M. D., Rohrbeck, C. A. y Fantuzzo, J. W. (2006). A meta-analytic review of social, self-concept, and behavioral outcomes of peer-assisted learning. Journal of Educational Psychology, 98(4), 732-749. https://doi.org/10.1037/0022-0663.98.4.732

González González, C. S. (2014). Estrategias para trabajar la creatividad en la educación superior: Pensamiento de diseño, aprendizaje basado en juegos y en proyectos. Revista de Educación a Distancia, 40, 2-15. https://revistas.um.es/red/article/view/234291/180001 
http://doi.org/10.15359/ree.25-1.1

http://www.una.ac.cr/educare

educare@una.ac.cr

González, G. y Díaz Matajira, L. (2005). Aprendizaje colaborativo: Una experiencia desde las aulas universitarias. Educación yeducadores, 8, 21-44. https://educacionyeducadores.unisabana. edu.co/index.php/eye/article/view/564/655

Huberman, A. M. y Miles, M. B. (2000). Métodos para el manejo y el análisis de datos. En C. A. Denman y J. A. Haro (Comps.), Por los rincones. Antología de métodos cualitativos en la investigación social (pp. 253-300). El Colegio de Sonora.

Hunt, D. P., Haidet, P., Coverdale, J. H. y Richards, B. (2003). The effect of using team learning in an evidence-based medicine course for medical students. Teaching and Learning in Medicine, 15(2), 131-139. https://doi.org/10.1207/S15328015TLM1502 11

Johnson, D. W. y Johnson, R. T. (1999). Making cooperative learning work. Theory into Practice, 38(2), 67-73. https://doi.org/10.1080/00405849909543834

Johnson, D. W., Johnson, R. T. y Smith, K. A. (2014). Cooperative learning: Improving university instruction by basing practice on validated theory. Journal on Excellence in College Teaching, 25(3-4), 85-118.

León del Barco, B. (2002). Elementos mediadores en la eficacia del aprendizaje cooperativo: Entrenamiento en habilidades sociales y dinámicas de grupo [Tesis Doctoral]. Universidad de Extremadura, Cáceres.

León del Barco, B., Felipe Castaño, E., Iglesias Gallego, D. y Marugán de Miguelsanz, M. (2014). Determinantes en la eficacia del aprendizaje cooperativo. Revista de Investigación Educativa, 32(2), 411-424. https://doi.org/10.6018/rie.32.2.172721

Masran, N.y Azizi, N. S. H. A. (2018). Effects of cooperative learning (STAD) on student achievement in Jawi among year five pupils. Advanced Science Letters, 24(7), 5334-5337. https://doi. org/10.1166/asl.2018.11729

Mendo-Lázaro, S., León-del Barco, B., Felipe-Castaño, E., Polo-del Río, M.-I. e Iglesias-Gallego, D. (2018). Cooperative team learning and the development of social skills in Higher Education: The variables involved. Frontiers in Psychology, 9, 1-11. https://doi.org/10.3389/ fpsyg.2018.01536

Munir, M. T., Baroutian, S., Young, B. R. y Carter, S. (2018). Flipped classroom with cooperative learning as a cornerstone. Education for Chemical Engineers, 23, 25-33. https://doi. org/10.1016/j.ece.2018.05.001

Ovejero, A. (1990). El aprendizaje cooperativo. Una alternativa eficaz a la enseñanza tradicional. PPU.

Putpuek, A. y Kiattikomol, P. (2017). Development of a blended online and offline learning model with think-pair-share collaborative learning and student team's achievement division competition. International Journal of Innovation and Learning, 22(2), 254-269. https://doi. org/10.1504/IJL.2017.085928 
http://doi.org/10.15359/ree.25-1.1

Puzio, K. y Colby, G. T. (2013). Cooperative learning and literacy. Journal of Research on Educational Effectiveness, 6(4), 339-360. https://doi.org/10.1080/19345747.2013.775683

Ravenscroft, S. P., Buckless, F. A., McCombs, G. B. y Zuckerman, G. J. (1995). Incentives in student team learning: An experiment in cooperative group learning. Issues in Accounting Education, 10(1), 97-109.

Reese, L., Balzano, S., Gallimore, R. y Goldenberg, C. (1995). The concept of education: Latino family values and American schooling. International Journal of Educational Research, 23(1), 57-81. https://doi.org/10.1016/0883-0355(95)93535-4

Reznitskaya, A., Anderson, R. C. y Kuo, L-J. (2007). Teaching and learning argumentation. The Elementary School Journal, 107(5), 449-472. https://doi.org/10.1086/518623

Šerić, M. y Praničević, D. G. (2018). Managing group work in the classroom: an international study on perceived benefits and risks based on students' cultural background and gender. Journal of Contemporary Management Issues, 23(1), 139-156. https://doi.org/10.30924/ $\underline{\mathrm{mj}} \mathrm{cmi} / 2018.23 .1 .139$

Slavin, R. E. (1978). Student teams and comparison among equals: Effects on academic performance and student attitudes. Journal of Educational Psychology, 70(4), 532-538. http://dx.doi.org/10.1037/0022-0663.70.4.532

Slavin, R. E. (1991). Student team learning: A practical guide to cooperative learning. (Developments in classroom instruction). National Education Association.

Slavin, R. E. y Oickle, E. (1981). Effects of cooperative teams on student achievement and race relations: Treatment by race interactions. Sociology of Education, 54(3) 174-180. https:// doi.org/10.2307/2112329

Soto Fernández, M. P. (2009). Desarrollo de las habilidades sociales en el aula. Revista Digital. Innovación y Experiencias Educativas, 14, 1-9. https://www.csif.es/contenido/andalucia/ educacion $/ 243960$

van Wyk, M.M. (2015). Measuring the effectiveness of student teams' achievement divisions as a teaching strategy on Grade 10 learners' economic knowledge. International Journal of Educational Studies, 10(2), 325-337. https://doi.org/10.1080/09751122.2015.11917664

Wanzek, J., Vaughn, S., Kent, S. C., Swanson, E. A., Roberts, G., Haynes, M., Fall, A.-M., StillmanSpisak, S. J. y Solis, M. (2014). The effects of team-based learning on social studies knowledge acquisition in high school. Journal of Research on Educational Effectiveness, 7(2), 183-204. https://doi.org/10.1080/19345747.2013.836765

Yusuf, Y. Q., Natsir, Y. y Hanum, L. (2015). A teacher's experience in teaching with student teamsachievement division (STAD) technique. International Journal of Instruction, 8(2), 99-112. https://doi.org/10.12973/iji.2015.828a 